\title{
Complex plasma afterglow
}

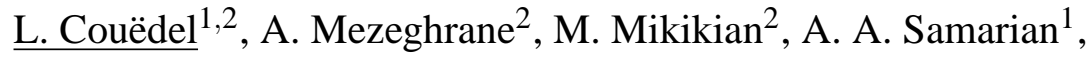 \\ M. Cavarroc ${ }^{2}$, Y. Tessier ${ }^{2}$ and L. Boufendi ${ }^{2}$ \\ ${ }^{1}$ School of Physics A28, The University of Sydney, NSW 2006, Australia \\ ${ }^{2}$ GREMI (Groupe de Recherches sur l'Énergétique des Milieux Ionisés), CNRS/Université \\ d'Orléans , 14 rue d'Issoudun, Orléans Cedex 2, France
}

\begin{abstract}
A numerical model for dusty plasma afterglow has been developed. Comparison with experimental data shows that the transition from ambipolar to free diffusion plays a major role in the decharging process
\end{abstract}

Dusty or complex plasmas are ionised gases composed of electrons, ions and neutral species in which macroscopic charged dust particles are immersed. The dust particle charge $\left(Q_{d}\right)$ is related to the ion and electron currents ( $I_{i}$ and $I_{e}$ respectively) onto the surface of the dust particle:

$$
\frac{d Q_{d}}{d t}=I_{e}+I_{i}
$$

These currents depend on the particle radius $r_{d}$, the electron (ion) density and the electron (ion) temperature [1]. At equilibrium, currents compensate each other. In laboratory plasma, these particles are negatively charged due to a higher mobility of the electrons. The dust particle charging process is thus highly dependent on plasma species densities and temperatures.

Many publications are devoted to the study of dust particle charge (see for example $[2,3,4]$ ). Recently the decharging of dust particles in complex plasma afterglow raises interest as well as the dust particle residual charge [5, 6, 7]. Indeed it has been observed that dust particles do keep residual electric charges when the power of a RF discharge is turned off. Moreover, negatively charged, neutral and positively charged dust particles have been obeserved in the late afterglow of the RF discharge. A four stage model has been proposed in order to explain these results (Fig.1)[6]:

- Firstly, the electron temperature decreases while the plasma density remains almost constant resulting in a strong decrease of dust particle charges.

- Secondly, the plasma density decreases but ion and electron densities remain equal leading to almost no change in particle charge. 

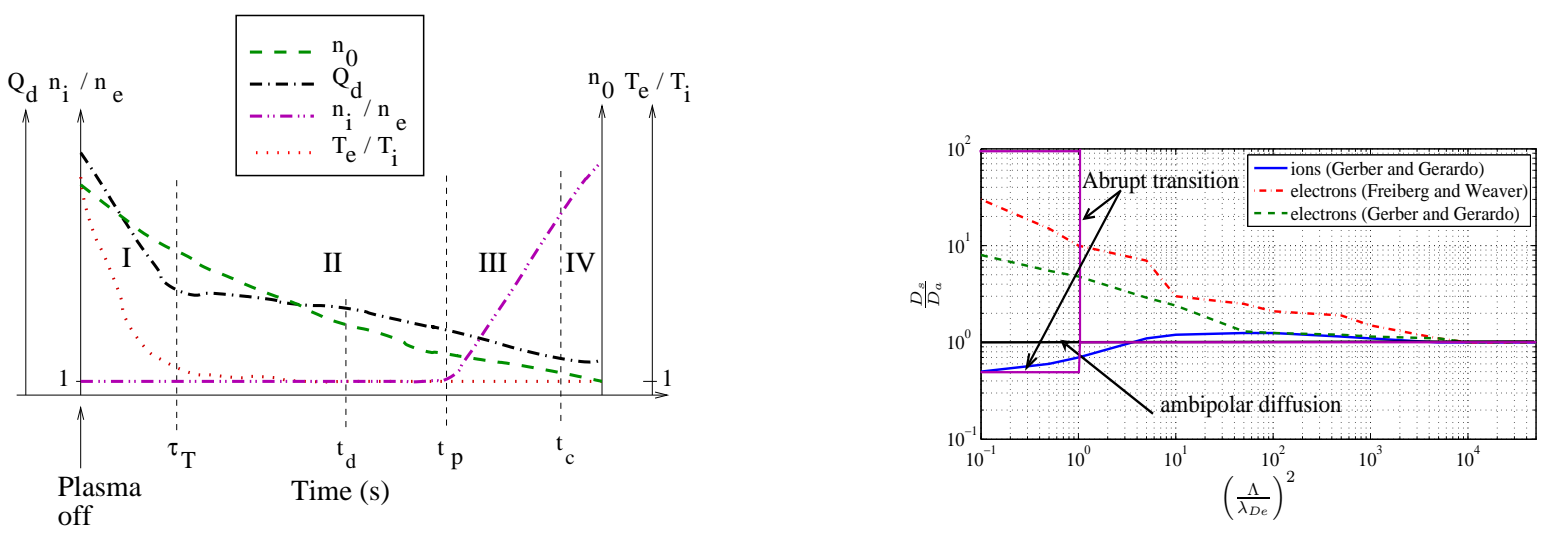

Figure 1: Left: Qualitative time evolution of dust charge, plasma density and electron temperature during the afterglow Four stages of the dust plasma decay can be identified: I - temperature relaxation stage up to $t_{T}$ II plasma density decay stage up $t_{p}$, III- dust charge volume stage $t_{c}$, IV - frozen stage.

Right: Evolution of the ratio $D_{s} / D_{a}(s=i, e)$ as a function of $\left(\Lambda / \lambda_{D e}\right)^{2}\left(D_{s}\right.$ is diffusion coefficient of the $s$ species and $D_{a}$ is the ambipolar diffusion coefficient). These data are extracted from Refs.[8] and [9].

- The third stage corresponds to the charge volume stage when the charge on dust particles can not be neglected anymore or when ion and electron densities are too small allowing large density differences to appear over distances less than the screening length. In both cases, this leads to a change in the diffusion regime, i.e. electrons and ions diffuse independently. As electron are running faster away than ions, the ratio $n_{i} / n_{e}$ becomes more than one resulting in a decrease of the dust particle charge.

- Finally, ion and electron densities are too small to influence the dust particle charges which can be considered as frozen (i.e. charging time tends to infinity).

This model predicts the existence of residual charges about $-3 e$ which is in agreement with the experimental mean residual charges but is unable to predict positive residual charges. Furthermore, influence of dust particles is taken into account in a very poor manner: the dust particles play a role only when their total charge volume is of the same order as the electron density which leads to an abrupt change from ambipolar to free diffusion of ions and electrons.

It has been shown that diffusion of charged species in a dust-free plasma afterglow deviates from ambipolar diffusion as soon as the ratio $\left(\Lambda / \lambda_{D e}\right) \sim 100$ where $\Lambda$ is the diffusion length and $\lambda_{D e}$ is the electron Debye length. $[9,10]$. These results indicate that electrons and ions are lost at different rates very soon in the decay process (Fig.1).

Consequently, a numerical model taking into account the transition from ambipolar to free diffusion has been developped: plasma losses due to diffusion onto the wall of the reactor are simulated using a OD fluid model in the same way as in a dust free plasma. The transition from ambipolar to free diffusion is simulated using either experimental results from Gerber 



Figure 2: Decay of an argon plasma at $P=0.4 m b a r$. From the left to the right: Electron temperature relaxation; density evolution; evolution of diffusion time.
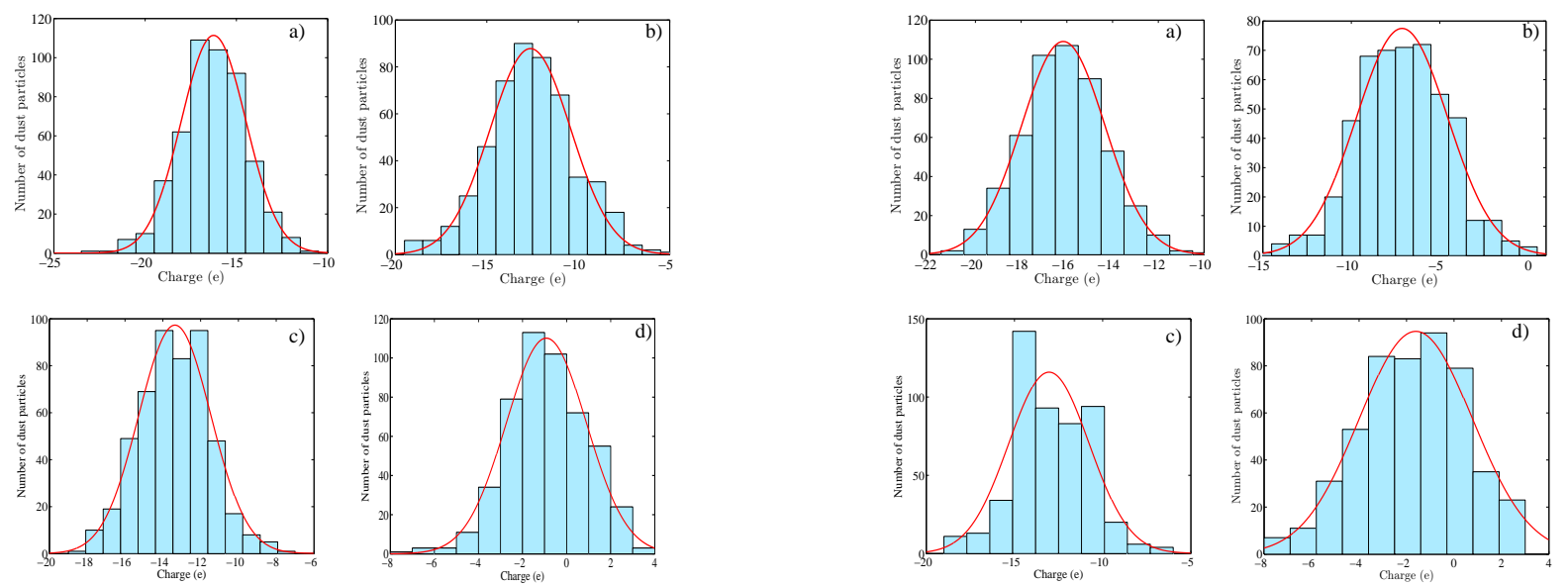

Figure 3: Numerical results for $190 \mathrm{~nm}$ radius dust particles with argon pressure $P=0.4$ mbar $(P=$ 0.3 Torr)(Left) or $P=1.2 \mathrm{mbar}\left(P=0.9\right.$ Torr) (Right) and $n_{d}=5 \cdot 10^{4} \mathrm{~cm}^{-3}$. a) Ambipolar diffusion until the end of the decay process. b) Abrupt transition from ambipolar to free diffsusion when Havnes parameter $P_{H}=0.5$. c) Using data from Gerber and Gerardo for transition from ambipolar to free diffusion [8]. d) Using data from Freiberg and Weaver for transition from ambipolar to free diffusion [9]

and Gerardo [8] or experimental results from Freiberg and Weaver [9] (the first ones point out a slower transition from ambipolar to free diffusion than the second ones). The dust particle charge as well as the plasma losses due to recombination onto the surface of dust particle are computed trough a modified Cui and Goree algorithm [11].

The initial electron temperature is taken as $T_{e 0}=3 e V$ and the ion temperature is supposed to be equal to the neutral temperature $T_{i}=T_{n}=T=0.03 \mathrm{eV}$. The diffusion length is taken $\Lambda=1 \mathrm{~cm}$ which is approximately the diffusion length of the PKE-Nefedov reactor in which experiments on residual dust charge have been performed [5, 6].

It can be seen in Fig.2, that the electron density deviates from the ion density very soon in the decay process. This effect is very important for the dust particle residual charges. Indeed the speed of the transition influences strongly the mean residual charge of dust particles (see Fig.3). 
For a pressure $P=0.4 \mathrm{mbar}(P=0.3$ Torr $)$, the simulated final dust distributions (when the charging time becomes much bigger than the plasma diffusion time (typically $\sim 10$ times in our simulation) are presented in Fig.3. The residual charge is $Q_{d_{\text {res }}} \simeq-16 e$ when no transition in the diffusion process is taken into account. When using a model based on an abrupt transition from ambipolar to free diffusion when the Havnes parameter $P_{H}=0.5$, the residual charge is smaller (in absolute value) $Q_{d_{\text {res }}} \simeq-13 e$ but still far from experimental value. It can be seen that the way the transition from ambipolar to free diffusion is taken into account has a great influence on the final dust charge distribution. Indeed, using Gerber and Gerardo data, the mean residual charge $Q_{d_{r e s}} \simeq-13 e$ whereas it is $Q_{d_{r e s}} \simeq-1 e$ using Freiberg and Weaver data. Furthermore, in the first case, there is no tail in the positive region of the dust particle charge distribution as there is one in the second case. The second residual charge distribution is similar to the experimental one [6].

For a pressure $P=1.2$ mbar $(P=0.9$ Torr $)$, the simulated final dust particle charge distributions are presented in fig.3. The residual charge is $Q_{d_{\text {res }}} \simeq-16 e$ when no transition in the diffusion process is taken into account. When using a model based on an abrupt transition from ambipolar to free diffusion when the Havnes parameter $P_{H}=0.5$, the residual charge is smaller (in absolute value) $Q_{d_{\text {res }}} \simeq-7 e$ but still far from experimental value. A dependance on the ambipolar to free diffusion transition is again seen with $Q_{d_{\text {res }}} \simeq-14 e$ when using Gerber and Gerardo data and $Q_{d_{r e s}} \simeq-2 e$ when using Freiberg and Weaver data which is close to experimental results [6].

The decharging process of dust particles is strongly dependent on the transition from ambipolar to free diffusion as their charges depend on the ratio $n_{i} / n_{e}$. It has been shown that the speed of transition influences the final residual dust charge distribution in a strong manner. The faster the transition occurs, the smaller the mean dust particle residual charge and a positive charge tail can even be obtained.

\section{References}

[1] S. Vladimirov, K. Ostrikov, and A. Samarian. Physics and Applications of Complex Plasmas (Imperial Press, London, 2005).

[2] C. Arnas, M. Mikikian, and F. Doveil. Phys. Rev. E, 60, 7420 (1999).

[3] T. Matsoukas and M. Russell. J. Appl. Phys., 77, 4285 (1995).

[4] A. A. Samarian and S. V. Vladimirov. Phys. Rev. E, 67, 066404 (2003).

[5] A. Ivlev, M. Kretschmer, M. Zuzic, et al.. Phys. Rev. Lett., 90, 055003 (2003).

[6] L. Couëdel, M. Mikikian, L. Boufendi, et al.. Phys. Rev. E, 74(2), 026403 (2006).

[7] L. Couëdel, A. Mezeghrane, M. Mikikian, et al.. J. Electrostatics, In press (2007).

[8] R. A. Gerber and J. B. Gerardo. Phys. Rev. A, 7(2), 781-790 (1973).

[9] R. J. Freiberg and L. A. Weaver. Phys. Rev., 170, 336-341 (1968).

[10] M. A. Gusinow and R. A. Gerber. Phys. Rev. A, 5(4), 1802-1806 (1972).

[11] C. Cui and J. Goree. IEEE Trans. Plasma Sci., 22, 151 (1994). 